\title{
Missing Pilgrims Tracking System Using GPS, GSM and Arduino Microcontroller
}

\author{
Zahida Parveen \\ Computer Science and Software Engineering dept. CCSE \\ $\mathrm{UoH}$ \\ Hail, Saudi Arabia \\ z.malikuoh@gmail.com
}

\author{
Kawther A.Aldhlan \\ Computer Science and Software Engineering dept. CCSE \\ UoH \\ Hail, Saudi Arabia \\ K_aldhlan@hotmail.com
}

\begin{abstract}
This research proposed centralize controlling system that can help to track missing pilgrims during Hajj season by using the wide spread GPS, GPRS and Arduino UNO Microcontroller. The system under study is planned to provide the facility to pilgrims, Ministry of Hajj and the law and peace enforcement in tackling with this issue. Similar applications related to this issue were developed and can be divided into two types from the perception of the device used for location determination. Some applications are using handheld Global Positioning System (GPS) receiver and others are using mobile phones with embedded GPS receiver. This paper proposed system that is designed in such a way to deal with both solutions at the same time. This gives more agility to the system to suite diverse user categories in term of age i.e. children, young and old people with some limitations. In the proposed system the location of the pilgrim is sent instantly to the web server in the form of General Packet Radio Service (GPRS) packet through the Global System for Mobile (GSM) network on demand. The system will also provide the facility to keep a check on a missing pilgrim for regular location updates after identifying that particular pilgrim.
\end{abstract}

Keywords-: Missing Pilgrims; Global Positioning System; uino Microcontroller; Pilgrims Tracking .

\section{INTRODUCTION}

Hajj is a holy virtues of Islam that is performed by very able Muslim once in a life, it is an annual performing virtue that can only be perform in Kingdom of Saudi Arabia. Every year during season of Hajj, millions of Muslims gathered in Holy place Makah to perform Hajj from all over the world. Among this huge number $70 \%$ of the pilgrims are foreigners (Table I).

During the hajj season the cases of missing and lost people are seen daily many pilgrims got separated from their group and cannot find their way back. In 2006 in one of the most known conference more than 2,500 cases of missing people in the area of Masjid al-Haram, in Makah were reported [Fakkar,2006]. In 2011 around 30,000 pilgrims were reported as lost during different activities. In record figures compared to previous years, 7998 pilgrims were reported missing in 2013 [Ministry of interior Kingdom of Saudi Arabia, Tuesday 15 October 2013]. Official figures on missing people during Hajj cannot be found however, it would be much more than the reported number. In the table mentioned above, most of Hajj pilgrims' are from different countries so are not familiar with the surroundings in Makah, so there are more chances of getting lost. Most of such cases are solved by the authorities and the Hajj guides but still the chances of trouble for the missing pilgrims and their family who are searching them are high. Missing pilgrims' cases are not infrequent and numerous traditional and technological tracking and searching systems have been introduced, but still a better solution is needed to solve this critical problem. There are many factors that affect the implementation and practical use of those systems like cost, methods and uncommon plate form.

The main objective of this research is to propose Missing pilgrims tracking system that will be activated just when a missing pilgrim is reported as missing. The system will provide a centralize availability of information by using latest technology of GPS (Global Positioning System), GSM (Global System for Mobiles) and Arduino UNO microcontroller. The system is aimed to utilize the latest and low cost technology for improving the process of Hajj.

TABLE I. HAJJ STATISTICS FOR LAST FIVE YEARS

\begin{tabular}{|c|c|c|c|}
\hline Year & Saudi Pilgrims & Foreign Pilgrims & Total \\
\hline $1430 / 2009$ & 154,000 & $1,613,000$ & $2,521,000$ \\
\hline $1431 / 2010$ & 989,798 & $1,799,600$ & 2.8 million \\
\hline $1432 / 2011$ & $1,099,523$ & $1,828,196$ & $2,927,717$ \\
\hline $1433 / 2012$ & $1,408,642$ & $1,752,931$ & $3,161,573$ \\
\hline $1434 / 2013$ & 600,718 & $1,379,531$ & $1,980,249$ \\
\hline
\end{tabular}




\section{LITERATURE REVIEW}

Similar applications related to this issue were proposed and developed in the last few years not only in Kingdom of Saudi Arabia but also in different other Muslim countries. The review of some of such systems is listed below.

One of the most widely recognized hardware being utilized now a day is RFID (Radio Frequency Identification) chip. A system to identify the pilgrims using smart chips (RFID) was developed by the students of King Fahd University of Petroleum and Minerals in 2006.The University managed the work of the Center for Computing and Communications, They propose a solution for this issue that was based on RFID technology. Their proposed system was design to help the Hajj authorities in the identification of pilgrims. The system was also helpful in controlling the crowd. They developed a prototype Pilgrim Identification System that utilizes a wristband RFID tag, an RFID reader and Graphical User Interface application as front end. A wristband that has RFID tag worn by a pilgrim, the RFID tag was suppose to store the hajji's personal information, his/her medical record and possibly E-purse. The complete flow of work of RFID system for pilgrim identification has been shown in Fig.l

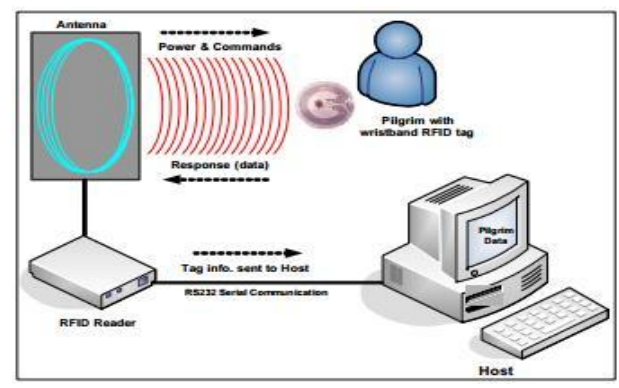

Fig.1. RFID Pilgrim Identification System

The figure clearly explained the working of the system that can work just on a limited small area i.e. the distance between RFID tag and RFID reader antenna must be very small.

After reviewing the system it was emerged that RFID technology has short range of action this limitation makes it not suitable for tracking the movement of pilgrims over large area. This system is useful just to identify the unrecognized pilgrims in a crowded environment and helping the authorities in managing the movement of pilgrims from the main checkpoints so cannot help in a situation when we want to look for missing pilgrims whose location is not identified. [1]

Another system that was developed to Track the pilgrims by the group leader (guide) using GPS and GPRS that is not only embedded in mobile systems but also can be embed in handheld devices like (wristbands). This gives more flexibility to the system to suite diverse user categories in term of age i.e. children, young and old people with some limitations. In this system the guide of Hajj group can monitor the assigned group of pilgrims through a web based application using various portable devices (Smart phone, iPad or laptop). The location of the pilgrim is sent instantly to the web server in the form of General Packet Radio Service (GPRS) packet through the Global System for Mobile (GSM) network, where it is saved in a Mobile Object Database (MOD).

The review of this system showed that the system is good and can be utilized but it's a distributed system i.e. each group of pilgrims is controlled by a leader having the internet enabled system and can just monitor his own group, what about the people who are not grouped and if we want to search a specific hajji from a centralized location. [2]

Another pilot program with the same objective was presented by a team of female students in the Department of Computer Science, Faculty of Computer and Information Sciences at University of Imam Muhammad bin Saud. The aim of the project was to keep track of pilgrims during the Hajj and Umrah using wireless sensor networks.

Beside that another system was develop by the students of University of Technology Malaysia having the same purpose of people tracking using Global Positioning System and Global System for Mobile Communication technology and Google Maps. The system used GPS, GSM modem for tracking purpose and Arduino UNO microcontroller for navigation purpose. The GPS provided information about the real time location of the people and sends the coordinate to the GSM modem through the microcontroller. Microcontroller used as the crossing point between GPS receiver and GSM modem. After receiving the location data, the data is sent receiving GSM modem through Short Message Service. The received coordinates sent to the control unit through the serial port. After the data processing, the location of the user can be viewed on Google Maps. The system also updates the user coordinate every 1 minute 10 second. Review of the above discussed system revealed that it was a good and cheap system but it continuously provides the information about the location of a person that is not necessary in tracking the pilgrims where we just want to track a person on demand when we need to know about his or her location it will also save the power utilization. [3]

\section{PROPOSED SYSTEM}

\section{A. System objectives}

- Help to determine the place of missing people quickly.

- $\quad$ Reduce the number of missing hajji.

- To reduce police men and guides effort for locating missing pilgrims. 


\section{B. System Overview}

The major goal of this research is to help the government of Saudi Arabia to find missing pilgrims easily and faster. In the research GPS is the main hardware component that is used GPS is a satellite-based navigation system that is consist of 24 satellites placed into orbit. The position determined by GPS is extremely accurate because of their parallel multi-channel design. The GPS will be very helpful and easy way not just to find missing pilgrims, but also to determine the location of undefined persons after hajj.

Missing pilgrims system is utilizing of GPS receiver, GPRS modem, Arduino UNO Microcontroller devices as hardware and Arduino, visual studio and Google Map as software. Each Pilgrim should own the GPS receiver which can be embedded in mobile phone .In the case of old people and children they may be don't have mobile phones in this case GPS bracelet tracker well be distributed to them. The cost of these bracelets will be paid by the users. When any missing pilgrim is reported the Passport Number or the GPS ID will be provided to the system through GUI form and send the request for location to through Arduino UNO Microcontroller and GPRS Modem which will enable you to receive the GPS data from the satellite and sends it to the web server that will be programmed to show the exact location on a Google map. Fig .2 illustrates the basic idea of the proposed system as a flow chart.

The flow chart of proposed approach gives an idea about the flow of data and information. The proposed approach starts work when there is a request for finding the location of a missing pilgrim the proposed system takes the ID (Passport Number, GPS ID, or Mobile Number) of missing pilgrim and pass on that ID to GPS Modem. Arduino UNO controller supposes to be act as intermediate between GPS Modem and GPS receiver. The request for location is send to the GPS receiver from the microcontroller after verifying the state of microcontroller and GPS. The coordinates (latitude and longitude) of the Hajji will send to the microcontroller which will be responsible for send the latitude and longitude in a proper format to the GUI. The GUI will be programmed to utilize this information to show the exact location on Google map as a final output.

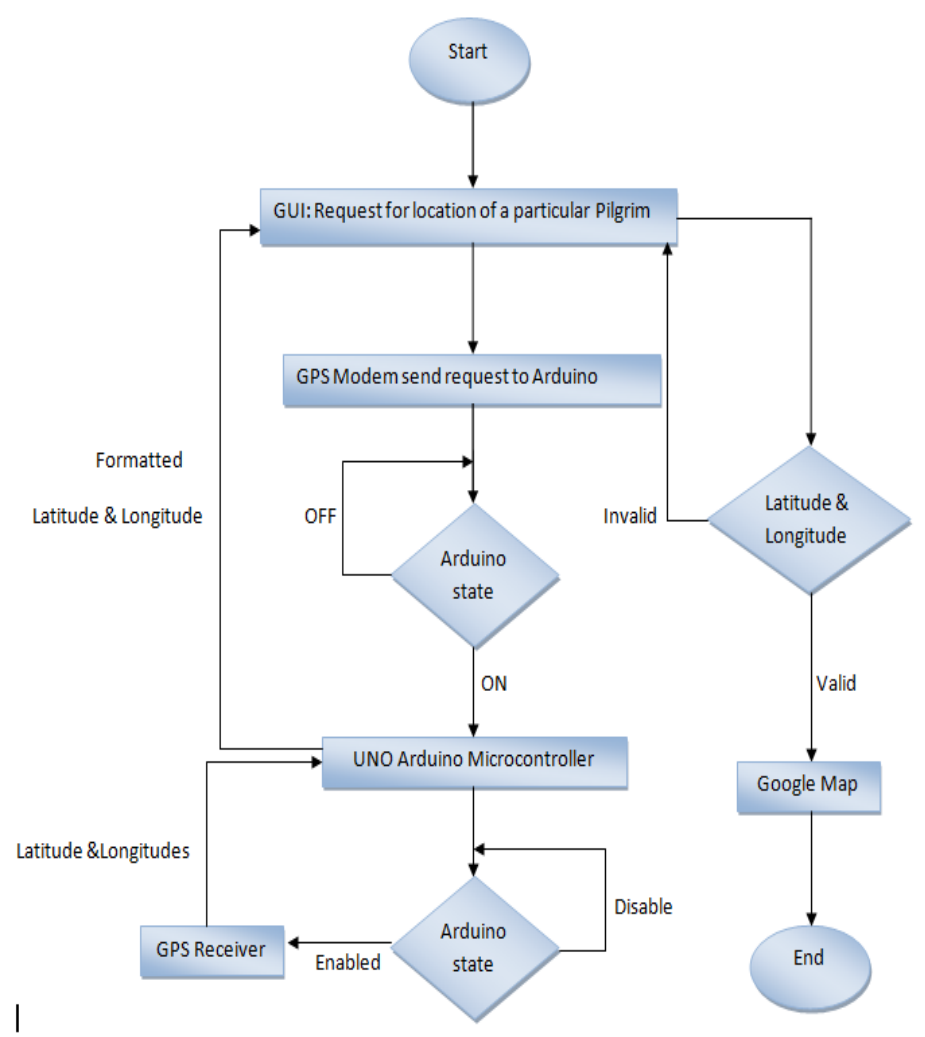

Fig-2 MPTS Flowchart

\section{Requirements for Tracking System}

The main requirements for the tracking system are:

- GPS Receiver

- GPS/GPRS Modems

- Arduino UNO Microcontroller

- Google Maps

- Internet access

\section{Hardware Tracking Devices}

A tracking device is an electronic card/tab that can be used to screen the location of an object or people by using the radio signal or satellite signal. In order to make our system more efficient and cost effective it proposed to use the GPS and Arduino UNO Microcontroller for exact and fast data retrieval. The minimum requirement of tracking devices is a GPS receiver that can send the latitude and longitude data to the Arduino UNO Microcontroller, a GPS modem that can receive location information from Arduino UNO Microcontroller and a Arduino UNO Microcontroller on which we can write the desire program to send the location request to GPS receiver. This device designed In order to be used to track pilgrim's by mobile phones with embedded GPS receiver and GPS Bracelet [4]. 


\section{E. GPS Receiver}

The Global Positioning System (GPS) is a satellite-based tracking system consists of network of 24 satellites present in the orbit. GPS is a universal navigation system that can work in all weather and geographical condition without any time or other constraints. A GPS enabled wristband and cell phone is shown in Fig.3. [5]

In proposed system the GPS receiver aimed to be used as embedded in a mobile system or in a wristband that will directly communicate with Arduino and GPRS Modem after receiving a location request from one of them.

\section{F. GPS/GPRS modem}

It is used to enable most devices to transmit data to various networks. It is facilitate a faster transmission of data over a GPRS connection. A GSM modem is shown in Fig.4. In proposed system GPRS Modem is used as intermediate between the Arduino UNO and Graphical User Interface and will be controlled by AT commands.[7]

The importance of the quality of cellular network cannot be ignored when studying about the working of GPRS, The studies reveled that in quality of services reliability is very crucial in any communication system and wireless networks should be optimized for excellent reliability to ensure system permanence. It is observed that the frequent rate of signal outages can be minimized by allocating power in a manner that each mobile has an extra signal to interference ratio (SIR. Therefore, power control is very essential when managing wireless communication systems. [6]

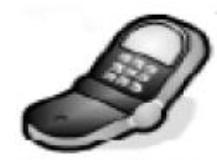

GPS Enabled Cell

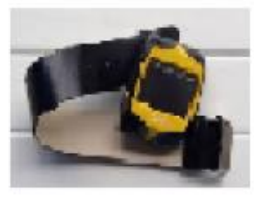

GPS Enabled

Wristband
Fig. 3. GPS Receivers

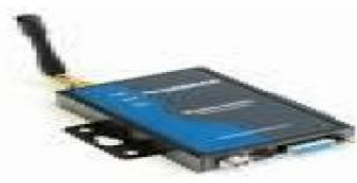

Fig.4. GSM/GPRS Modem

\section{G. Arduino Microcontroller}

It is taking inputs from a different switches or sensors, and controlling different functions. Arduino projects can work alone, or can be communicate with any other software of one's interest. It's an open-source hardware board. [4]

In the proposed approach The Arduino UNO microcontroller is preferred to choose The Uno board will be programmed for sending the on demand request for location with specified ID and receive the location information.

\section{FRAME WORK OF PROPOSED APPROACH}

The architecture framework of the system shows the complete working flow of the system as shown in Fig-5. The request for the location of a missing Pilgrim is send from the GUI of the proposed system to the GPRS Modem and through UNO Microcontroller which send the request to GPS receiver .GPS receiver send the requested latitude and longitude to the Arduino microcontroller which will responsible for decoding the data into required format and send it to GPRS Modem from where it will be taken as input by the GUI forms and show the exact location of the required person on a Google map.

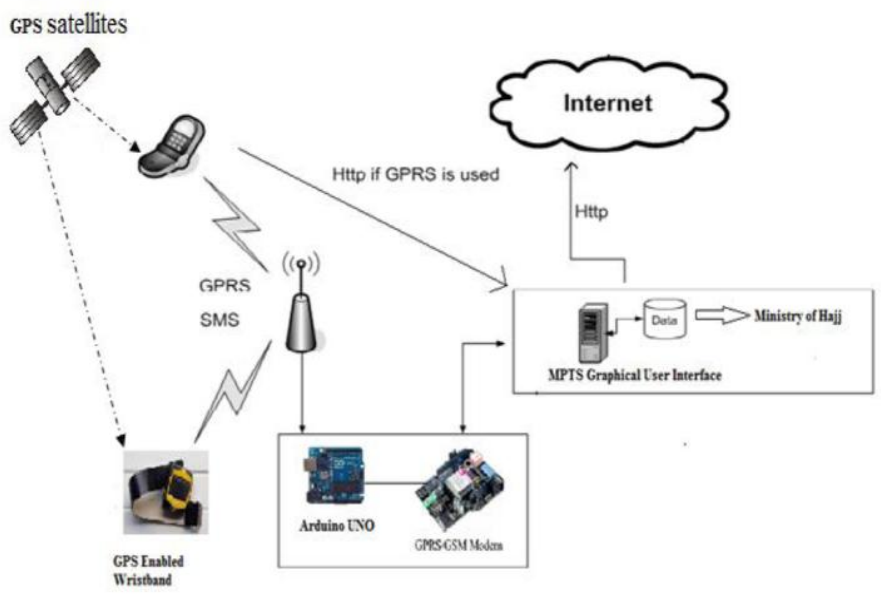

Fig. 5. Missing P ilgrim Tracking System Architecture

\section{INTEGRATED GUI AND DATABASE}

The proposed system is planned to design a web based application for user interaction that will be act as interface between user and the system. All the inputs and outputs will send to and received from the hardware components will be carried out through this interface.

A very simple look of interface as a prototype is provided here just as an idea likely to be modified is shown in Fig-6. 


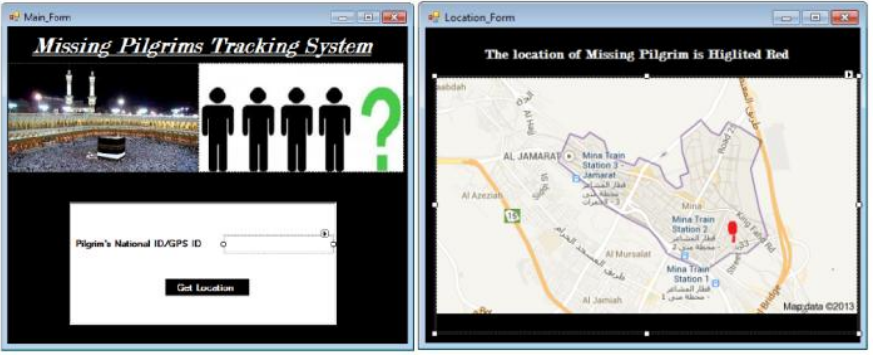

Fig-6 Initial prototype of the system

It is proposed that the system will utilizes the database from Ministry of hajj to get the complete information of all registered Pilgrims but that database will be modified according to the system requirements i.e. will add a new column for saving the GPS receiver unique ID and any other related information.

\section{CONCLUSIONS\& RECOMMENDATIONS}

Finding the missing pilgrims is already a big issue which is still unresolved. The purpose of this research is to provide a service to Islamic religion to cope up with this problem. The proposed approach tends to use the minimum resources and provide the maximum utility with less effort it is aimed as this approach will be helpful and useful for pilgrims and government as well. The GPS is great inventions we wish use it in a good way to reduce the number of missing people and the fatigue of people who works in this filed.
It is suggested as a future work to add a $\operatorname{SOS}$ (Save Our Soul) button to the GPS receiver so that in case of any pilgrim get lost and could not find the way back or in case of any emergency can send the alarm message to the head office for help. This system can also be combined with RFID system to provide more facilities to the pilgrims and government authorities.

\section{References}

[1] Mohammed Mohandes, Maan Kousa, Ahmed A Hussain, "An RFID Based Pilgrim Identification System”,2007

[2] Aladdein, Amro, and Abdel-Muti Nijem Qasem, Prof. "Pilgrims Hajj Tracking System (e-Mutawwif)" 2012 ed. Vol. 5.

[3] People Tracking System using Global Positioning System and Global System for Mobile Communication 2013 ed. University of Teknologi Malaysia.

[4] Banzi, M. "Started with Arduino" (2nd Edition). Make: Books. 2011.

[5] "GPS/AGPS chip set targets telematics", navigation apps Electronic Engineering Times, 2004. (1336): 86-86.

[6] http://www.researchgate.net/publication/51018297 QoS Reliability in GPRS Systems ARTICLE in MODERN APPLIED SCIENCE · APRIL 2011 DOI: 10.5539/mas.v5n2p57 · Source: DOAJ

[7] Hasan, K.S., Rahman, M., Haque, A.L., Rahman, M.A., Rahman, T. and Rasheed, M.M., Cost effective GPS-GPRS based object tracking system.In Proceedings of the International MultiConference of Engineers andComputer Scientists 2009 (Hong Kong, March 18 - 20, 2009) 